\title{
The LncRNA, the phosphatase, and the macrophage: can they team up for support in inflammatory diseases?
}

\author{
Marcel Kuhny", Michael Huber ${ }^{\#}$ \\ Institute of Biochemistry and Molecular Immunology, Medical Faculty, RWTH Aachen University, Aachen, Germany \\ \#These authors contributed equally to this work. \\ Correspondence to: Prof. Dr. Michael Huber. Institute of Biochemistry and Molecular Immunology, RWTH Aachen University, Pauwelsstr. 30,52074 \\ Aachen, Germany. Email: mhuber@ukaachen.de. \\ Comment on: Han X, Huang S, Xue P, et al. LncRNA PTPRE-AS1 modulates M2 macrophage activation and inflammatory diseases by epigenetic \\ promotion of PTPRE. Sci Adv 2019;5:eaax9230.
}

Submitted Apr 29, 2020. Accepted for publication May 11, 2020.

doi: 10.21037/atm-2020-111

View this article at: http://dx.doi.org/10.21037/atm-2020-111

Macrophages are immune cells of the myeloid lineage comprising a heterogeneous group of cells distributed in various tissues (e.g., Kupffer cells in the liver, microglial cells in the central nervous system, osteoclasts in the bone, and alveolar macrophages in the lung). They are endowed with a remarkable plasticity in response to (micro-) environmental signals, hence, significant phenotypic changes can be the consequence $(1,2)$. The initial classification into classically activated macrophages (M1) and alternatively activated macrophages (M2) has evolved into a more meaningful division into classically activated macrophages, woundhealing macrophages, and regulatory macrophages, with the latter two being associated with the M2 type (1). This classification, however, is still too strict and fails to represent the (patho-) physiological variety of existing types of macrophages. For instance, tumour-associated macrophages (TAMs) share characteristics of regulatory as well as woundhealing macrophages whereas adipose tissue macrophages (ATMs), exerting wound-healing characteristics in nonobese humans, can acquire qualities of classically activated macrophages in obesity (1). Furthermore, there are striking similarities between M2 type activation and the induction of endotoxin tolerance in macrophages (3). Nevertheless, the initial M1/M2 classification, is still in use for simplicity reasons, particularly in studies homing in on the dichotomy of macrophages and their resulting biochemical and cell biological distinctions. This was done, for instance, in the present study by Han et al., who treated bone marrowderived macrophages (BMDMs) either with LPS or IL-4 to activate them towards a M1 or M2 phenotype, respectively.

The authors' aim was to identify new long non-coding RNAs (LncRNAs), which determine the polarization of macrophages. To this end, they screened specifically for antisense-coded LncRNAs upregulated in IL-4- but downregulated in LPS-treated macrophages. One of these LncRNAs was the, until this point unassigned, PTPRE-AS1.

Using sophisticated knock-down, knock-out, and overexpression techniques in BMDMs and the RAW264.7 murine macrophage cell line, the LncRNA PTPRE-AS1 was convincingly shown to act as a repressor of IL-4-stimulated M2 macrophage activation through enhanced expression of the tyrosine phosphatase PTPRE. This was demonstrated elaborately by the analysis of expression of typical M2 markers, such as IL-10, ARG1, and YM1, as well as IL-4induced activation of the mitogen-activated protein kinases ERK1/2.

The activation of the MAPK pathway comprising the consecutive kinases RAF, MEK1/2, and ERK1/2 appears to control the activation of M2-associated genes (4). Particularly ERK1/2 phosphorylate respective transcription factors. The strength of the IL-4-induced expression of M2associated genes correlated with the strength of activation of ERK1/2, and both negatively correlated with expression of PTPRE-AS1 and PTPRE. PTPRE can be expressed as a transmembrane (memPTPRE) and as a cytoplasmic phosphatase (cytPTPRE). While memPTPRE is highly expressed in brain, testes, and lymph nodes, cytPTPRE can be found in spleen, thymus, and lung. Similar to most 
receptor type tyrosine phosphatases, memPTPRE and cytPTPRE contain two cytoplasmic tyrosine phosphatase domains (5). In a previous publication, heterologous overexpression of PTPRE attenuated phorbol ester-induced ERK1/2 activation in a phosphatase activity-dependent manner (6). This raises the interesting question of which target protein is dephosphorylated by PTPRE to mediate its regulation of $\mathrm{M} 2$-associated genes. With PTPRE being a tyrosine phosphatase, the MAP kinases are most likely not direct substrates of PTPRE. IL-4 receptor-proximal signal transduction crucially depends on tyrosine phosphorylation events with Janus kinases (JAKs) and the large adaptor protein IRS-2 taking on a central role. Inhibition of JAKs by PTPRE should not only affect downstream ERK1/2 activation, but also STAT6 activation (7). The latter, however, has been excluded by Han et al. Thus, IRS-2 would be a candidate worth studying, since this adaptor protein can be involved in a plethora of regulatory protein interactions, with PTPRE being a potential direct or indirect protein partner. Therefore, the determination of the phosphotyrosine interactome of IRS-2 in IL-4stimulated BMDMs from WT, PTPRE-AS1 KO, and PTPRE KO mice should be an important analytical step to decipher the molecular basis of the functional interaction between PTPRE and the MAPK pathway, as well as the role of ERK1/2 activation with respect to M2 macrophage activation. Interestingly, the role of PTPRE seems to be receptor- and/or cell type-specific. E.g., PTPRE-deficient murine bone marrow-derived mast cells stimulated via the high affinity receptor for $\operatorname{IgE}(\mathrm{F} c \varepsilon R I)$ clearly revealed that PTPRE negatively controlled tyrosine phosphorylation of receptor-proximal spleen tyrosine kinase (SYK) and of the adaptors linker of activated T cells (LAT) and SH2-domaincontaining leukocyte protein of $76 \mathrm{kDa}$ (SLP76), but had no effect on ERK1/2 activation, when compared to WT cells (8).

Next, the authors speculated that the level of PTPRE expression might determine the development and severity of M1 and M2 polarized diseases. To address this, they made use of the acute, dextran sulphate sodium (DSS)-induced colitis mouse model first. DSS is a sulphated polysaccharide that is directly toxic to the colonic epithelium, thus causing severe inflammation in the colon associated with weight loss and increased occurrence of diarrhoea and bleeding (summarized as "disease activity index"). The comparison of WT and PTPRE-AS1 KO mice intriguingly revealed an attenuated disease activity index in KO compared to WT mice, suggesting that an increased expression of
M2-associated genes might counteract the DSS-induced degenerative inflammatory reaction. Indeed, IL-4 treated colon macrophages purified from PTPRE-AS1 KO animals revealed reduced expression of PTPRE, enhanced expression of M2-associated genes such as Arg1 and $Y m 1$, and increased activation of ERK1/2, when compared to macrophages purified from DSS-treated WT animals as corroborated by RT-qPCR analysis of colon tissue samples. It was also noted that the levels of PTPRE-AS1 and PTPRE were significantly attenuated in WT mice with DSS-induced colitis. Hence, reduction of PTPRE-AS1 and PTPRE may polarize macrophages toward a M2 response in the course of DSS-induced colitis, thus mitigating the severity of colitis development.

Strengthening the IL-4-induced production of M2associated genes through targeting PTPRE or PTPRE$A S 1$ expression might be a meaningful approach for the treatment of additional inflammatory conditions. However, macrophages of Ptpre-deficient mice exhibit defects in regulating the respiratory burst, which might be problematic in the course of bacterial infections (9). Thus, tissue-specific macrophage-targeting would be required not to harm the patient.

The authors' findings further suggested that PTPRE$A S 1$ and PTPRE might also be involved in modulating the severity of $M 2$ type inflammatory diseases. To prove this, they made use of a mouse model of M2-associated, cockroach extract (CRE)-induced pulmonary allergic inflammation. The prediction was that by enabling a stronger activation of $\mathrm{M} 2$-associated genes, the $\mathrm{KO}$ of PTPRE-AS1 should result in a more devastating pulmonary disease compared to WT animals. Indeed, CRE-treated PTPRE-AS1 KO mice displayed significantly augmented total numbers of inflammatory cells in the bronchoalveolar fluid, compared to WT mice. Particularly, the numbers of eosinophils and macrophages were enhanced in the bronchoalveolar fluid, as well as the recruitment of inflammatory cells to the lungs was exacerbated in CREtreated KO animals. Similar to the DSS-induced colitis model, expression of PTPRE-AS1 and PTPRE was reduced upon challenge with CRE in WT mice, indicating that the reduced PTPRE axis might promote the M2 nature of the allergic disease, and suggesting that in PTPREAS1 KO animals, the induction of M2 type genes should be even stronger. Certainly, expression of M2 type genes, such as $A r g 1$ and $Y m 1$, as well as ERK1/2 activation were augmented in IL-4-stimulated lung macrophages purified from allergic PTPRE-AS1 KO mice compared to allergic 
WT mice. This was corroborated in respective lung tissue samples. Thus, PTPRE-AS1 still allows the development of an adequate response in allergic pulmonary inflammation, however, it simultaneously protects from a detrimental course of the disease as can be observed in PTPRE-AS1deficient animals.

Intriguingly, hepatitis $\mathrm{C}$ virus ( $\mathrm{HCV}$ ) has also found a way to downregulate PTPRE expression by means of a virus genome-derived small RNA (10). PTPRE levels were significantly attenuated in liver tissue and peripheral blood mononuclear cells from HCV-infected humans compared to uninfected controls. It is tempting to speculate that $\mathrm{HCV}$-mediated reduction of PTPRE in innate immune cells might drive development of $\mathrm{T}_{\mathrm{H}} 2$ allergic responses, since about $5 \%$ of patients with viral hepatitis develop skin rashes and urticarial reactions resembling mast cell-driven reactions (11). This is also in line with a reported negative role of PTPRE in the activation of mast cells during an allergic response (8).

Finally, the authors make a strong argument for an important role of PTPRE-AS1 and PTPRE in human allergic disorders as they found a correlation between patients with severe allergies and a reduced expression of PTPRE-AS1, PTPRE, and WDR5. This correlation might be strong enough to serve as a predictive clinical marker and PTPRE and PTPRE-AS1 consequently represent potential targets for intervention in the treatment of severe allergic diseases. However, the bench-to-bedside transition of these results, i.e., the development of small molecule inhibitors, will require more verification in humans. Though human and murine alternatively activated macrophages are expected to be highly conserved, several prominent differences in gene expression are already known which might culminate to differences in the regulation of an allergic response. A prominent example pertains to the prototypic alternative activation marker ARG1 (12). Although a homolog exists in humans, ARG1 induction is confined mainly to murine macrophages. In addition, YM1 and FIZZ1, measured in the murine models by Han et al., lack homologs in humans. At the same time, human alternatively activated macrophages express certain markers, which are absent in mice e.g., the nucleotide G proteincoupled receptor GPR105 (12). With respect to chemokine production, IL-4 upregulates a group of six chemokines in human macrophages (CCL13, CCL14, CCL17, CCL18, CCL22, and CCL24), of which CCL14, CCL18, and CCL23 lack murine orthologs; CCL17 and CCL24 do have murine orthologs, however, are exclusively up-regulated in humans. Interestingly, chemokines produced by both species target a conserved set of chemokine receptors (CCR1, CCR2, CCR3, and CCR4) and, by extension, similar cellular infiltrates in both species (12). In light of all these inter-species differences, it will be a future task to reevaluate the molecular functions and regulatory activities of the PTPRE-AS1, PTPRE, WDR5, and ERK1/2 cluster in humans.

Mechanistically, PTPRE-AS1 was demonstrated by Han et al. to directly interact with WDR5, a core subunit of the histone H3 Lys4 (H3K4) methyltransferase complexes, MLL and SET1 (13), and to mediate H3K4 trimethylation of the PTPRE promoter region, hence epigenetically activating the expression of the PTPRE gene. To this end, PTPRE-AS1 recruits WDR5 to the PTPRE promoter enhancing PTPRE expression, and eventually allowing the regulation of IL-4-induced M2 macrophage activation by interfering with ERK1/2 activation.

In recent years, LncRNAs have been recognized to regulate transcription of inflammatory genes. LncRNAs directly interact with upstream signalling proteins acting as decoys or modulate post-translational modifications, serve as scaffolds for the assembly of ribonucleoprotein complexes, or guide histone modifiers to specific loci. Consequently, LncRNAs like LincRNA-EPS broadly suppress transcription of inflammatory genes, thereby maintaining homeostasis of resting macrophages. Others enhance or suppress transcription of specific inflammatory genes, e.g., THRIL acts as a scaffold for hnRNPL to enhance TNF- $\alpha$ transcription, while LincRNA-p21 blocks transcription of Rela, coding for the NFкB p65 subunit. Beyond the spectrum of broad vs. specific control of gene expression, LncRNAs enable time-dependent regulation,

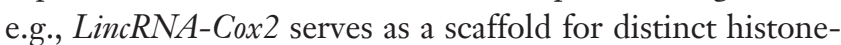
modifying complexes during early and late phase of the inflammatory response (14).

A prime example for the LncRNA-enforced control of an inflammatory response is the switch of the acute LPS response to the chronic response pattern found in endotoxin tolerant innate immune cells. A host of LPS-induced LncRNAs have been found to suppress expression of inflammatory genes. Many of these LncRNAs directly target

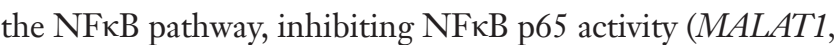
LincRNA-p21), preventing the activation of IKK (NKILA), or the ubiquitination of TRAF6 (Mirt2). Others (SeT, Lnc$I L-17 R, I L 7-A S)$ repress the transcription of NFкB target genes (15). Underscoring the similarities between endotoxin tolerant and alternatively activated M2 macrophages, 
LincRNA-Cox2 was also found to simultaneously inhibit functions of M1 macrophages, while enhancing M2 macrophage-dependent proliferation and survival of hepatocellular carcinoma cells (16). Other LncRNAs have been found to skew macrophage polarization by either promoting or suppressing gene transcription. E.g., IL-4dependent M2 polarization is promoted by LncRNA-XIST, but inhibited by NIFK-AS1 $(17,18)$.

The striking finding that PTPRE-AS1 alone exerts profound control over M2 polarization indicates that this LncRNA is a pivotal checkpoint in the activation and polarization of macrophages. Albeit PTPRE-AS1 clearly regulates the expression of PTPRE, it is noteworthy that knock-down of the LncRNA more potently enhanced M2 polarization than knock-down of PTPRE. The explanation might lie in the interaction of PTPRE$A S 1$ with WDR5, a component of several histonemodifying complexes. Binding of LncRNAs to WDR5 confers chromatin localization, which stabilizes WDR5 and maintains transcriptionally active chromatin (19). Consequently, the PTPRE-AS1:WDR5 complex may modify the epigenetic markers of more genes, other than PTPRE, relevant for M2 polarization. Also, there may be competition of different LncRNAs interacting with WDR5, which in turn would be sequestered from other chromatin sites by binding to PTPRE-AS1. In this regard, future experiments may elucidate if upregulation of PTPRE$A S 1$ and PTPRE promote the expression of M1-specific genes while simultaneously disfavouring M2 polarization of macrophages.

As stated in the beginning of this article, macrophages are cells endowed with an extreme plasticity and an ability to precisely react to their environment. The prototypical alternatively activated macrophage does not exist and depending on the environmental situation, wound-healing macrophages and regulatory macrophages might easily switch to a TAM-like or an ATM-like phenotype $(1,2)$. Hence, it would be important to study the impact of different environmental factors on PTPRE-AS1 and PTPRE, but also how PTPRE-AS1 and PTPRE might influence the plasticity of the diverging sub-groups of alternatively activated macrophages. This seems of special importance as LncRNAs, which control the M1/M2 polarization, have a major impact on cancer development. E.g., the M2promoting $L n c R N A-X I S T$ has been associated with lung cancer while the M1-promoting LncRNA-COX2 prevents immune evasion and metastasis of hepatocellular carcinoma.

In summary, PTPRE-AS1 expression might be a promising clinical marker while targeting PTPRE-AS1 or PTPRE by gene-therapy could represent a versatile way to skew the macrophage polarization to the benefit of a yet to be determined spectrum of patients.

\section{Acknowledgments}

The authors thank Dr. Natalia Rinis for critically reviewing this manuscript.

Funding: None.

\section{Footnote}

Provenance and Peer Review: This article was commissioned and reviewed by the Academic Editor Xiuhua Yao, MD (Tianjin Neurosurgical Institute, Tianjin Huanhu Hospital, Tianjin, China).

Conflicts of Interest: Both authors have completed the ICMJE uniform disclosure form (available at http://dx.doi. org/10.21037/atm-2020-111). The authors have no conflicts of interest to declare.

Ethical Statement: The authors are accountable for all aspects of the work in ensuring that questions related to the accuracy or integrity of any part of the work are appropriately investigated and resolved.

Open Access Statement: This is an Open Access article distributed in accordance with the Creative Commons Attribution-NonCommercial-NoDerivs 4.0 International License (CC BY-NC-ND 4.0), which permits the noncommercial replication and distribution of the article with the strict proviso that no changes or edits are made and the original work is properly cited (including links to both the formal publication through the relevant DOI and the license). See: https://creativecommons.org/licenses/by-nc-nd/4.0/.

\section{References}

1. Mosser DM, Edwards JP. Exploring the full spectrum of macrophage activation. Nat Rev Immunol 2008;8:958-69.

2. Murray PJ, Wynn TA. Protective and pathogenic functions of macrophage subsets. Nat Rev Immunol 2011;11:723-37.

3. Pena OM, Pistolic J, Raj D, et al. Endotoxin tolerance represents a distinctive state of alternative polarization (M2) in human mononuclear cells. J Immunol 2011;186:7243-54. 
4. Neamatallah T. Mitogen-Activated Protein Kinase Pathway: A Critical Regulator in Tumor-associated Macrophage Polarization. J Microsc Ultrastruct 2019;7:53-6.

5. Liang J, Shi J, Wang N, et al. Tuning the Protein Phosphorylation by Receptor Type Protein Tyrosine Phosphatase Epsilon (PTPRE) in Normal and Cancer Cells. J Cancer 2019;10:105-11.

6. Wabakken T, Hauge H, Finne EF, et al. Expression of human protein tyrosine phosphatase epsilon in leucocytes: a potential ERK pathway-regulating phosphatase. Scand J Immunol 2002;56:195-203.

7. Tanuma N, Shima H, Nakamura K, et al. Protein tyrosine phosphatase epsilonC selectively inhibits interleukin-6and interleukin-10-induced JAK-STAT signaling. Blood 2001;98:3030-4.

8. Akimoto M, Mishra K, Lim KT, et al. Protein tyrosine phosphatase epsilon is a negative regulator of FcepsilonRImediated mast cell responses. Scand J Immunol 2009;69:401-11.

9. Sully V, Pownall S, Vincan E, et al. Functional abnormalities in protein tyrosine phosphatase epsilondeficient macrophages. Biochem Biophys Res Commun 2001;286:184-8.

10. Bhattarai N, McLinden JH, Xiang J, et al. Hepatitis C virus infection inhibits a Src-kinase regulatory phosphatase and reduces T cell activation in vivo. PLoS Pathog 2017;13:e1006232.

11. Marone G, Rossi FW, Detoraki A, et al. Role of superallergens in allergic disorders. Chem Immunol Allergy 2007;93:195-213.

Cite this article as: Kuhny M, Huber M. The LncRNA, the phosphatase, and the macrophage: can they team up for support in inflammatory diseases? Ann Transl Med 2020;8(22):1536. doi: 10.21037/atm-2020-111
12. Martinez FO, Helming L, Gordon S. Alternative activation of macrophages: an immunologic functional perspective. Annu Rev Immunol 2009;27:451-83.

13. Bochyńska A, Lüscher-Firzlaff J, Lüscher B. Modes of Interaction of KMT2 Histone H3 Lysine 4 Methyltransferase/COMPASS Complexes with Chromatin. Cells 2018. doi: 10.3390/cells7030017.

14. Mathy NW, Chen XM. Long non-coding RNAs (lncRNAs) and their transcriptional control of inflammatory responses. J Biol Chem 2017;292:12375-82.

15. Vergadi E, Vaporidi K, Tsatsanis C. Regulation of Endotoxin Tolerance and Compensatory Antiinflammatory Response Syndrome by Non-coding RNAs. Front Immunol 2018;9:2705.

16. Ye Y, Xu Y, Lai Y, et al. Long non-coding RNA cox-2 prevents immune evasion and metastasis of hepatocellular carcinoma by altering M1/M2 macrophage polarization. J Cell Biochem 2018;119:2951-63.

17. Sun Y, Xu J. TCF-4 Regulated lncRNA-XIST Promotes M2 Polarization Of Macrophages And Is Associated With Lung Cancer. Onco Targets Ther 2019;12:8055-62.

18. Zhou YX, Zhao W, Mao LW, et al. Long non-coding RNA NIFK-AS1 inhibits M2 polarization of macrophages in endometrial cancer through targeting miR-146a. Int J Biochem Cell Biol 2018;104:25-33.

19. Subhash S, Mishra K, Akhade VS, et al. H3K4me2 and WDR5 enriched chromatin interacting long non-coding RNAs maintain transcriptionally competent chromatin at divergent transcriptional units. Nucleic Acids Res 2018;46:9384-400. 